\title{
Selecting appropriate control variables for a heat-integrated distillation system with prefractionator
}

\author{
H. K. Engelien, S. Skogestad \\ Department of Chemical Engineering, Norwegian University of Science and Technology, 7491 Trondheim, Norway
}

\begin{abstract}
A heat-integrated prefractionator arrangement is studied for a ternary separation of a propane-butane-pentane mixture. These types of integrated prefractionator arrangements have large energy savings compared with the best of the direct or indirect sequence with no heat integration. However, a heat-integrated distillation system can be more difficult to control than a non-integrated arrangement, so good control systems are essential. In this work, the focus is on selecting control variables that will ensure that the promised energy savings are achieved. The method of self-optimizing control (Skogestad, 2000) has been used to provide a systematic procedure for the selection of controlled variables, based on steady state economics. The results show that the integrated prefractionator arrangement can have large energy savings compared with non-integrated arrangements and that controlling the ratio of the distillate flow to the feed flow has good self-optimizing properties.
\end{abstract} (C) 2004 Elsevier Ltd. All rights reserved.

Keywords: Heat-integrated; Distillation columns; Ternary separation; Multi-effect; Control

\section{Introduction}

Distillation is an energy consuming process that is used for about $95 \%$ of all fluid separation in the chemical industry and accounts for an estimated 3\% of the world energy consumption (Hewitt, Quarini, \& Morell, 1999). However, there are potentials for improvement and heat integration of distillation columns can be a method for achieving large savings.

For ternary separations there are three classical separation schemes: direct split, indirect split (IS) and the prefractionator arrangement. Energy savings can be achieved for these separation schemes by running one of the columns at a higher pressure and integrating the condenser of the high-pressure (HP) column with the reboiler of the low-pressure (LP) column. For these "multi-effect" systems there are two modes of integration: forward integration, where the heat integration is in the direction of the mass flow, and backward integration, where the integration is in the opposite direction of the mass flow.

In this study, the focus is on finding self-optimizing control variables for a prefractionator arrangement with forward integration. These type of integrated arrangements have been shown to have large energy savings compared with both

E-mail address: skoge@chembio.ntnu.no (S. Skogestad). integrated and non-integrated arrangements, see for example Cheng and Luyben (1985), and Emtir, Rév, and Fonyó (2001). In the literature there are however, only a few articles concerned with the optimization and control of these integrated arrangements.

Ding and Luyben (1990) presented control studies of a direct split system and a backward integrated prefractionator systems for ternary separation of benzene-toluene-xylene. Two cases were considered: a low and a high purity requirement for the products. For the integrated prefractionator arrangement, there is control of the two impurities in the sidestream (from main column) by controlling the sidestream flowrate and the sidestream draw-off tray. For the low-purity case they found that dynamically the prefractionator arrangement is about the same as the conventional direct split configuration. For the high purity case both systems are controllable, but the direct split configuration gives much better load rejection. They also found that the control of the sidestream toluene composition was particularly poor.

Bildea and Dimian (1999) also investigated the controllability for a system with a prefractionator and a sidestream main column. For the forward and backward integrated case they looked at four different designs, depending on the light/heavy split in the prefractionator. The authors concluded that in general the forward heat integration scheme is the easiest to control. 
Emtir et al. (2003) compared five different energyintegrated schemes, among them the forward and backward integrated prefractionator arrangement, with a non-integrated direct split sequence. The study compared the total annual costs (TAC) and the controllability of the different schemes. In terms of TAC they found that the backward integrated direct split configuration has the maximum savings of $37 \%$. The integrated prefractionator arrangements have similar savings of $34 \%$ for the forward-integrated case and $33 \%$ for the backward-integrated case. In terms of controllability, the authors found that the integrated direct and indirect split arrangements was similar to the non-integrated direct split. The more complex configurations were found to be more difficult to control due to interactions between the control loops. They also found that in terms of controllability that the forward-integrated schemes were preferred.

In this work, a heat-integrated arrangement consisting of a prefractionator and a main column with sidestream is studied for the ternary separation of a propane-butane-pentane solution. The objective of the work has been on the selection of controlled variables, that is, finding which variables that should be controlled so that the energy savings can be achieved. The concept of self-optimizing control (Skogestad, 2000), which normally is based on steady state economics, is used to provide a systematic framework for the selection of the controlled variables. This method involves a search for the variables that, when kept constant, indirectly lead to near-optimal operation with acceptable economic loss. In self-optimizing control, rather than solving the optimization problem on-line, the problem is transferred into a simple feedback problem (Skogestad, 2000). In practice, this means that when the plant is subject to disturbances it will still operate within an acceptable distance from the optimum and there is no need to re-optimize when disturbances occur.

\section{The integrated prefractionator arrangement}

The system studied in this paper is a ternary separation of propane-butane-pentane using a forward multi-effect heat-integrated scheme. The separation is carried out in two columns operating at different pressures (see Fig. 1). The first column is a high pressure prefractionator, which performs the propane/pentane split. Both the distillate and the bottom product from the high-pressure column are fed to the second, low-pressure column. Here propane, butane and pentane are the products from the distillate, sidestream and bottom stream, respectively. The top part of the LP column (above sidestream) performs the propane/butane split while the bottom part (below the sidestream) performs the butane/pentane split. The heat integration between the two columns is in an integrated reboiler/condenser, where the condensing heat from the HP column is used to boil the LP column.

The forward heat integrated scheme was selected for this study because it was found previously by other authors to

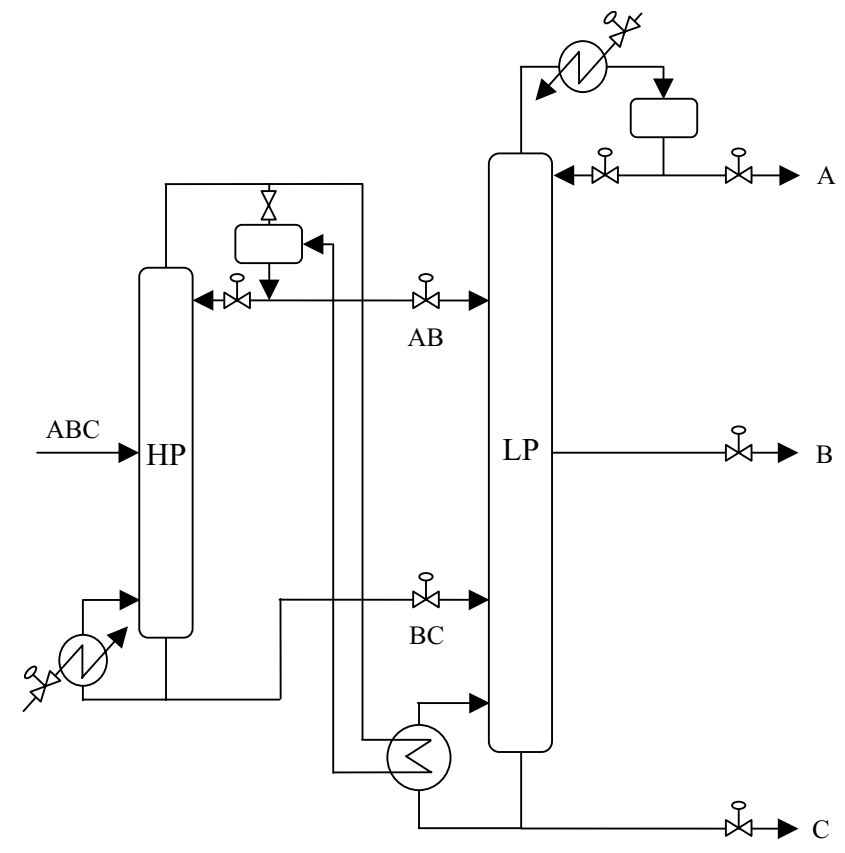

Fig. 1. The forward heat-integrated prefractionator arrangement.

be the most easy to control (Bildea \& Dimian, 1999; Emtir et al., 2003). For a backward integrated system the prefractionator would be at a low pressure while the main column would be at the high pressure. The integrated condenser would then be between the top of the main (HP) column and the bottom of the prefractionator (LP). The energy savings assuming content relative volatility would be identical (see Table 2).

The feed data and column specifications are given in Table 1.

\subsection{Energy savings for integrated prefractionator arrangements}

For a thermally coupled distillation column (Petlyuk), the energy savings can be typically $30 \%$ compared with the direct or indirect split arrangement (Halvorsen, 1999). However, using a prefractionator which is not only thermally coupled, but also heat integrated with the columns run at different pressures, can have savings of around $50 \%$ or more compared to the best of the direct or indirect sequence (Ding \& Luyben, 1990).

Minimum vapour flowrate calculations for ternary mixtures can be done using the Underwood equations for

Table 1

Feed data and column specifications
Feedrate $(F)$

Feed composition $\left(z_{\mathrm{F}}\right)$

Feed liquid fraction

Number of stages

Relative volatilities
$300 \mathrm{~mol} / \mathrm{s}$

Propane 0.15 , butane 0.70 , pentane 0.15 $q=1$ (all columns)

HP column: 20, LP column: 40

Propane 7.98, butane 3.99 , pentane 1.00 


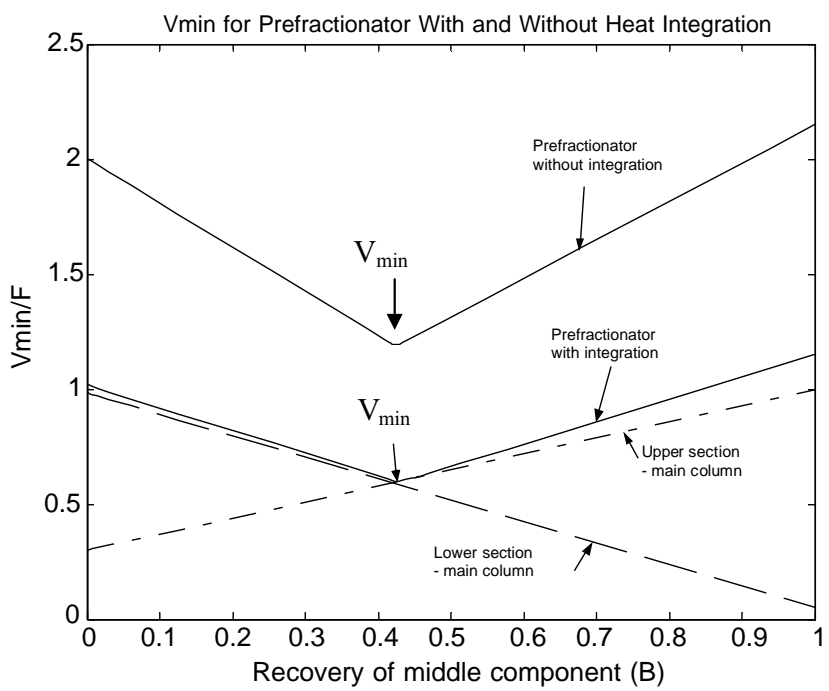

Fig. 2. Comparing $V_{\min }$ for prefractionator arrangement with and without integration.

minimum vapour flowrate for sharp splits and constant relative volatility. In the prefractionator, the minimum vapour flowrate as a function of the recovery of the middle component, $r_{\mathrm{B}, \mathrm{D}}$, is calculated from

$V_{\min , 1}=\left(\frac{\alpha_{\mathrm{A}} z_{\mathrm{A}}}{\alpha_{\mathrm{A}}-\theta}+\frac{\alpha_{\mathrm{B}} r_{\mathrm{B}, \mathrm{D}} z_{\mathrm{B}}}{\alpha_{\mathrm{B}}-\theta}\right) F$

The Underwood roots, $\theta_{\mathrm{A}}$ and $\theta_{\mathrm{B}}$ are found from the feed equation (King, 1980). The break in the prefractionator curve in Fig. 2 is the point where we go from using $\theta_{\mathrm{A}}$ to using $\theta_{\mathrm{B}}$ in Eq. (1). The vapour flowrate in the second column is calculated from Kings equation (King, 1980) for binary mixtures. Note that the effect of pressure on the separation is not included in these formulas.

For both the prefractionator arrangements, the energy saving is dependent on the recovery of the middle component from the prefractionator. Using the Underwood equation for the prefractionator and Kings equation for the upper and lower part of the main column we can plot the minimum vapour flowrate as a function of the recovery of the middle component from the prefractionator. A sharp split between $\mathrm{A} / \mathrm{C}$ in the prefractionator and $\mathrm{A} / \mathrm{B}$ and $\mathrm{B} / \mathrm{C}$ in the main column has been assumed. Fig. 2 shows a comparison of the minimum vapour flowrate required for an integrated and a non-integrated prefractionator arrangement as a function of the recovery of the middle component (further detail is given in Engelien, 2004). From the figure it can be seen that in this case both the integrated and non-integrated system has rather sharp optimums. This is not normally the case for the non-integrated scheme, but occurs for the feed composition and relative volatility used in the example, because the recoveries of the "preferred split" and the "balanced split" happen to be the same (see for example, Christiansen, 1997). Normally, this does not happen, and the non-integrated prefractionator system has a flat optimum, between the "preferred split" and the "balanced split". For the integrated system the optimum is always sharp, as seen in Fig. 2.

Table 2 is showing comparisons between energy requirements for integrated and other non-integrated schemes, based on the minimum vapour flowrate expressions described above (sharp splits). The energy savings are given as percentage improvement compared to the best of the non-integrated direct or indirect sequence. In all cases the integrated prefractionator arrangements have higher energy savings compared to the other schemes. The highest savings occur when there is a high concentration of the middle component. For feed composition [0.150.70.15] the integrated prefractionator arrangements have savings of $70 \%$, compared to the best of the direct or indirect non-integrated sequence. In terms of energy the best of the other integrated schemes for this feed is a direct split arrangement with forward or backward integration, which has $49 \%$ savings.

Constant relative volatility has been used in all columns (see Table 1). Also, it is optimal for all the cases, except the indirect split, to use liquid feed $(q=1)$ to all the columns. The indirect split, the indirect split with backward integration (ISB) and the prefractionator arrangement with reverse integration $(\mathrm{PB})$ would require less vapour flow for the $\mathrm{A} / \mathrm{B}$ split if the feed to this section was vapour. However, for the two integrated cases this would result in a vapour being feed from a LP column to a HP column, something that would require compression. This would lead to added costs and has therefore not been considered as a viable option.

Table 2

Comparison of energy savings $\left(V_{\min }\right)$ for different feed compositions (compared to the best of the non-integrated direct or indirect sequence) for mixtures with constant relative volatility, $\alpha=\left[\begin{array}{lll}8 & 4\end{array}\right]$

\begin{tabular}{|c|c|c|c|c|c|c|c|c|}
\hline$z_{\mathrm{F}}$ & DS (\%) & IS (\%) & Petlyuk (\%) & DSF $(\%)$ & DSB $(\%)$ & ISF $(\%)$ & ISB $(\%)$ & $\mathrm{PF} / \mathrm{PB}(\%)$ \\
\hline$\left[\begin{array}{llll}1 / 3 & 1 / 3 & 1 / 3\end{array}\right]$ & -0.44 & 0.00 & 34.86 & 34.86 & 34.86 & 36.54 & 36.54 & 51.77 \\
\hline$\left[\begin{array}{llll}0.7 & 0.15 & 0.15\end{array}\right]$ & 0.00 & -4.05 & 13.87 & 13.87 & 13.87 & 14.11 & 14.11 & 21.65 \\
\hline$\left[\begin{array}{llll}0.1 & 0.45 & 0.45\end{array}\right]$ & -3.79 & 0.00 & 39.20 & 46.47 & 46.47 & 39.20 & 39.20 & 66.66 \\
\hline 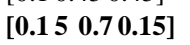 & -0.05 & 0.00 & 42.47 & 49.13 & 49.13 & 42.47 & 42.47 & 70.17 \\
\hline$\left[\begin{array}{llll}0.4 & 5 & 0.1 & 0.45\end{array}\right]$ & -1.29 & 0.00 & 20.85 & 20.85 & 20.85 & 22.00 & 22.00 & 28.89 \\
\hline$\left[\begin{array}{llll}0.15 & 0.15 & 0.7\end{array}\right]$ & -10.70 & 0.00 & 35.08 & 40.08 & 40.08 & 35.08 & 35.08 & 57.64 \\
\hline$\left[\begin{array}{llll}0.45 & 0.45 & 0.1\end{array}\right]$ & 0.00 & -2.41 & 31.83 & 31.83 & 31.83 & 32.22 & 32.22 & 49.02 \\
\hline
\end{tabular}

DS: direct split; DSF: direct split, forward integrated; DSB: direct split, backward integrated; IS: indirect split; ISF: indirect split, forward-integrated; ISB: indirect split, backward integrated; PF: prefractionator, forward integrated; PB: prefractionator, backward integrated; Petlyuk: thermally coupled columns. 
Vapour feed is therefore used only in the non-integrated indirect split case (columns at same pressure); for all the other cases liquid feed is considered to be the optimal operation. In the Petlyuk arrangement there is also vapour feed to the main column, but this is standard for this arrangement.

\section{Self-optimizing control}

The objective of the study is to implement a simple "optimal" control scheme for the integrated prefractionator arrangement by finding and controlling the variables in the system that will directly ensure optimal economic operation. Then, when there are disturbances in the system, there is no need to re-optimize.

The method of self-optimizing control involves a search for the variables that, when kept constant, indirectly lead to near-optimal operation with acceptable loss (Skogestad, 2000). The procedure consists of six steps: (1) a degree of freedom (DOF) analysis, (2) definition of cost function and constrains, (3) identification of the most important disturbances, (4) optimization, (5) identification of candidate controlled variables and (6) evaluation of loss with constant setpoints for the alternative sets of controlled variables.

The first important step in this systematic procedure is to analyse the number of degrees of freedom for the system. For the integrated prefractionator arrangement there are 11 dynamic (control) DOF, when assuming a fixed feedrate. These are: the boilup in the HP column, the condensation rate in the HP column, reflux, distillate and bottom flowrate from both columns, sidestream flowrate in the LP column, boilup in the LP column and condensation rate in the LP column (see Fig. 1).

For this distillation system there are four holdups in the reboilers and condensers that have to be stabilised, but these have no steady state effects and therefore no effects on the cost function. This then leaves seven degrees of freedom for optimization.

In the formulation of the objective function there are two 'conflicting' elements: to produce as much valuable product as possible, but using as little energy as possible. For a given feed, the cost function is defined as the amount of propane, butane and pentane from the LP column (at $0.99 \mathrm{~mol} \%$ or more) multiplied by the relevant product prices, minus the cost of the feed and the cost of boilup

$J=p_{\mathrm{D}} D+p_{\mathrm{S}} S+p_{\mathrm{B}} B-p_{\mathrm{F}} F-p_{\mathrm{V}} V$

From the total mass balance, we get

$J=\left(p_{\mathrm{D}}-p_{\mathrm{F}}\right) D+\left(p_{\mathrm{S}}-p_{\mathrm{F}}\right) S+\left(p_{\mathrm{B}}-p_{\mathrm{F}}\right) B-p_{\mathrm{V}} V$

Further, it has been assumed that the products have the same value, so $p_{\mathrm{D}}=p_{\mathrm{S}}=p_{\mathrm{B}}=p$ we have

$J=\left(p-p_{\mathrm{F}}\right)(D+B+S)-p_{\mathrm{V}} V=\left(p-p_{\mathrm{F}}\right) F-p_{\mathrm{V}} V$
With a given feedrate, $F$, the first term is fixed. Minimising $J$ is then equivalent to minimising

$J^{\prime}=-V$

where $V$ is the vapour flow in the bottom of the HP column and $Q_{\mathrm{B}, \mathrm{HP}}=V_{\mathrm{HP}} H_{\text {vap }}$.

Having defined the objectives, the system constraints must be defined. These are the model equations (equality constraints), i.e. the mass, component end energy balances for the distillation process and the operational constraints (inequality constraints). In addition to requiring positive flows, the following seven constraints have been specified:

- the pressure in the LP column should be greater than or equal to 1 bar;

- the pressure in the HP column should be less than or equal to $15 \mathrm{bar}$;

- the reboiler duty in the LP column $\left(Q_{\mathrm{B}, \mathrm{LP}}\right)$ must equal the condenser duty in the HP column $\left(Q_{\mathrm{C}, \mathrm{HP}}\right)$ (equality constraint);

- the product purity of the distillate $\left(x_{\mathrm{AD}, \mathrm{LP}}\right)$, side $\left(x_{\mathrm{BS}, \mathrm{LP}}\right)$ and bottom stream ( $\left.x_{\mathrm{CB}, \mathrm{LP}}\right)$ should be above or equal to $99 \mathrm{~mol} \%$;

- the area in the combined reboiler/condenser should be less than or equal to $A_{\max }$, where $A_{\max }$ is calculated from the optimal steady state solution assuming $\left(T_{\text {cond,HP }}-\right.$ $T_{\text {reb,LP }}=5^{\circ} \mathrm{C}$ and $U=906 \mathrm{~W} / \mathrm{m}^{2} \mathrm{~K}$. In practice the area constraint can, for example, be implemented by allowing the area to vary by using a bypass.

The optimization problem can then be formulated as: $\min \left(-J^{\prime}(x, u, d)\right)$, subject to: $g_{1}(x, u, d)=0$ (model equations) and $g_{2}(x, u, d) \leq 0$ (operational constraints). Here, $x$ are state variables, $u$ are independent variables that can be affected (DOF for optimization) and $d$ are independent variables that can not be affected (disturbances).

By solving the optimization problem we find the nominal steady state operating point, i.e. the optimal operating point for the multi-effect distillation when there are no disturbances. This gives us the optimal nominal values for all the variables in the system.

Then the most important disturbances in the system have to be defined. The optimization is then repeated for the various disturbances to find the active constraints and the objective value at each disturbance. The most important disturbance that has been considered is variations in the feed flow of $\pm 20 \%$ and variations in feed compositions $\left(z_{\mathrm{F}, \mathrm{B}}=\right.$ \pm 0.1 mol fraction). The optimal solution for the nominal case and for the disturbances can be seen in Table 3 .

\subsection{Active constraints}

The active constraints of the system are found from the optimization. For this system we would expect to find that the three product compositions, the pressure in the LP column and the area of the exchanger are active constraints. How- 
Table 3

Optimum solution for nominal case and for disturbances

\begin{tabular}{|c|c|c|c|c|c|}
\hline Operating case & $V_{\text {opt }}(\mathrm{mol} / \mathrm{s})$ & $x_{\mathrm{AD}, \mathrm{HP}}$ & $x_{\mathrm{BD}, \mathrm{HP}}$ & $r_{\mathrm{BD}, \mathrm{HP}}$ & $x_{\mathrm{BB}, \mathrm{HP}}$ \\
\hline Nominal & 159.31 & 0.37 & 0.61 & 0.349 & 0.76 \\
\hline$F+20 \%$ & 190.70 & 0.38 & 0.61 & 0.346 & 0.76 \\
\hline$F-20 \%$ & 127.75 & 0.37 & 0.61 & 0.351 & 0.76 \\
\hline$z_{\mathrm{F}, \mathrm{B}}+0.1$ & 162.29 & 0.23 & 0.75 & 0.405 & 0.84 \\
\hline$z_{\mathrm{F}, \mathrm{B}}-0.1$ & 154.33 & 0.52 & 0.47 & 0.300 & 0.68 \\
\hline$x_{\mathrm{CB}, \mathrm{HP}}$ & $x_{\mathrm{AD}, \mathrm{LP}}$ (light) & $x_{\mathrm{BS}, \mathrm{LP}}$ (middle) & $x_{\mathrm{CB}, \mathrm{LP}}$ (heavy) & $P_{\mathrm{HP}}($ bar) & $P_{\mathrm{LP}}($ bar $)$ \\
\hline 0.24 & 0.9999 & 0.99 & 0.99 & 7.4 & 1.0 \\
\hline 0.24 & 0.9999 & 0.99 & 0.99 & 7.6 & 1.0 \\
\hline 0.24 & 0.999985 & 0.99 & 0.99 & 7.2 & 1.0 \\
\hline 0.16 & 0.9999 & 0.99 & 0.99 & 6.0 & 1.0 \\
\hline 0.32 & 0.9999 & 0.99 & 0.99 & 8.8 & 1.0 \\
\hline$L_{\mathrm{T}, \mathrm{LP}}(\mathrm{mol} / \mathrm{s})$ & $L_{\mathrm{T}, \mathrm{HP}}(\mathrm{mol} / \mathrm{s})$ & $D_{\mathrm{HP}}(\mathrm{mol} / \mathrm{s})$ & $B_{\mathrm{HP}}(\mathrm{mol} / \mathrm{s})$ & $D_{\mathrm{LP}}(\mathrm{mol} / \mathrm{s})$ & $S_{\mathrm{LP}}(\mathrm{mol} / \mathrm{s})$ \\
\hline 273.6 & 40.4 & 119.9 & 180.1 & 45.0 & 211.7 \\
\hline 330.6 & 48.7 & 143.3 & 216.7 & 54.0 & 254.0 \\
\hline 217.4 & 32.2 & 96.4 & 143.6 & 36.0 & 169.3 \\
\hline 278.2 & 32.5 & 129.2 & 170.8 & 30.0 & 242.1 \\
\hline 264.9 & 42.5 & 115.4 & 184.6 & 60.0 & 181.2 \\
\hline$B_{\mathrm{LP}}(\mathrm{mol} / \mathrm{s})$ & $B_{\mathrm{HP}} / F$ & $Q_{\mathrm{B}, \mathrm{HP}}(\mathrm{MW})$ & $Q_{\mathrm{B}, \mathrm{HP}} / F(\mathrm{MW} /(\mathrm{mol} \mathrm{s}))$ & $Q_{\mathrm{B}, \mathrm{HP} / \mathrm{LT}, \mathrm{HP}}(\mathrm{MW} /(\mathrm{mols}))$ & $Q_{\mathrm{B}, \mathrm{HP} / \mathrm{LT}, \mathrm{LP}}(\mathrm{MW} /(\mathrm{mol} \mathrm{s}))$ \\
\hline 43.3 & 0.600 & 3.3736 & 0.0112 & 0.083 & 0.0123 \\
\hline 52.0 & 0.602 & 4.0335 & 0.0112 & 0.083 & 0.0122 \\
\hline 34.7 & 0.598 & 2.7086 & 0.0113 & 0.084 & 0.0125 \\
\hline 27.9 & 0.569 & 3.4219 & 0.0114 & 0.105 & 0.0123 \\
\hline 58.8 & 0.615 & 3.2931 & 0.0110 & 0.077 & 0.0124 \\
\hline$Q_{\mathrm{C}, \mathrm{HP}}(\mathrm{MW})$ & $Q_{\mathrm{B}, \mathrm{LP}}(\mathrm{MW})$ & $Q_{\mathrm{C}, \mathrm{LP}}(\mathrm{MW})$ & $T_{\mathrm{btm}, \mathrm{HP}}\left({ }^{\circ} \mathrm{C}\right)$ & $T_{\text {top }, \mathrm{HP}}\left({ }^{\circ} \mathrm{C}\right)$ & $A\left(\mathrm{~m}^{2}\right)$ \\
\hline-3.1942 & 3.1942 & -5.7292 & 75.55 & 40.02 & 821.87 \\
\hline-3.8173 & 3.8173 & -6.9156 & 76.70 & 41.00 & 821.87 \\
\hline-2.5660 & 2.5660 & -4.5562 & 74.38 & 39.04 & 821.87 \\
\hline-3.3417 & 3.3417 & -5.5414 & 63.91 & 40.25 & 821.87 \\
\hline-2.9882 & 2.9822 & -5.8411 & 86.12 & 39.70 & 821.87 \\
\hline
\end{tabular}

Active constants are shown in bold.

ever, for this problem only the following four constraints were active (see Table 3):

- the pressure in the LP column, which is equal to 1 bar;

- the product purity of the sidestream, which is equal to 0.99 ;

- the product purity of the bottom stream, which is equal to 0.99 ;

- the area in the integrated exchanger, which is equal to $A_{\max }$.

The pressure in the HP column and the product purity of the distillate were not active constraints.

When a variable is at a constraint then active constraint control is implemented, i.e. for this case $P_{\mathrm{LP}}, x_{\mathrm{BS}, \mathrm{LP}}, x_{\mathrm{CB}, \mathrm{LP}}$ and area $(A)$ will be controlled. For the area it is assumed that there is a bypass that can be manipulated in order to "control" the area and this should be kept "closed" to maximise $A$. This bypass will therefore be fully closed to maximise the area and thus the heat exchange between the two columns. Implementing the active constraint control means that we are left with two unconstrained degrees of freedom for which control variables have to be selected.
In this case, where the costs of the products are the same, it is the value of boilup that is minimised. In another case where the product prices are different, the purity constraint is likely to be active for the product(s) that has the highest value.

There may also be other cases when the active constraints change. Take, for instance, a case when there are changes in the feed composition, such that the condensation in the HP column is higher than the energy requirements of the LP column. In terms of Fig. 2 this means that the minimum vapour flowrate curve for the prefractionator will lie above the curve for the main column, for all recoveries of the middle component. In order to balance the columns it may then be optimal to overpurify the least valuable product in the main column, thus one purity constraint is no longer active. Another possible operation is that the prefractionator no longer performs a sharp $\mathrm{A} / \mathrm{C}$ split and the light component (A) will appear in the bottom stream in order to reduce the prefractionator duty. It is therefore important for the column operation and control system to study the feed composition and the effects of expected disturbances. 
If the condensation in the prefractionator is less than the boilup in the main column this can result in the purity requirements of the LP column not being met, or that we get pure $\mathrm{A}$ at the top, or pure $\mathrm{C}$ at the bottom of the prefractionator.

\subsection{Testing different control variables}

Above it was found that four of the constraints were active constraints, thereby leaving two degrees of freedom for self-optimizing control. This means that there are still two unconstrained variables that we need to control in order to specify the operation. To find out which variables are best suited we calculate the loss when different variables are fixed at their nominal optimal value (found from the optimization) when there are disturbances. Here it has been decided to keep the product concentration of the distillate stream at its minimum value of $99 \mathrm{~mol} \%$. This is justified since fixing the concentration in the distillate stream gives very low loss $(\leq 0.0013 \%)$. Thus there is little to gain by overpurifying this product and we may as well keep it at $99 \%$. This "active constraint" consequently leaves one degree of freedom.

For the remaining unconstrained degree of freedom a number of control variables were tested. The results from the loss calculation are presented in Table 4, where the extra cost of duty is presented as the percentage of the optimal duty requirement for each of the disturbances. Here, a $1 \%$ extra duty cost corresponds to about US\$ 25000 per year when assuming production of 330 days per year and the cost of energy at US\$ $0.062 \mathrm{MJ}^{-1}$.

From Table 4 it can be seen that constant setpoint control of many variables, like the pressure in the HP column $\left(P_{\mathrm{HP}}\right)$, result in large losses when there are disturbances in the feed flowrate or composition. Other variables result in infeasible operation for some of the disturbances. For most of the cases this is caused by the purity constraint not being reached in the sidestream of the main column.
It can also be seen that from the variables tested, the ratio of the distillate or the bottom stream in the prefractionator to the feed stream $\left(D_{\mathrm{HP}} / F, B_{\mathrm{HP}} / F\right)$ has the lowest loss. This indicates that both of these are good self-optimizing variables. If one of these variables is controlled at a constant setpoint then the system will be closer to the optimum energy savings than if, for example, the pressure was to be controlled. The implementation error $( \pm 10 \%)$ for $D_{\mathrm{HP}} / F$ was also tested and found to result in a loss of 2.9 and $20 \%$, respectively. The largest implementation error was for the case of $D_{\mathrm{HP}} / F=10 \%$. Including the implementation error this gives an average loss of $4.3 \%$ when controlling $D_{\mathrm{HP}} / F$.

\subsection{Proposed control structure for prefractionator arrangement}

A suggested control structure for integrated prefractionator arrangement using $D_{\mathrm{HP}} / F$ as the selfoptimizing control variable is presented in Fig. 3. The active constraint control loops are shown shaded. It should be noted that this is only a suggestion for the pairing of controlled and manipulated variables. The focus of the study is on identifying the control variables, based on steady state economics, and not on the pairing or dynamic issues.

The control structure has the following features:

(I) Stabilising level loops (four)

- the reflux flow $\left(L_{T, H P}\right)$ is used for level control in the HP condenser;

- the distillate flows $\left(D_{\mathrm{LP}}\right)$ is used for level control in the LP condenser;

- the bottom flowrate from the HP column $\left(B_{\mathrm{HP}}\right)$ is used to control the level in the HP column;

- the bottom flowrate from the LP column $\left(B_{\mathrm{LP}}\right)$ is used to control the level in the LP column.

(II) Active constraint control loops (four)

- the bottom product purity $\left(x_{\mathrm{C}}=x_{\mathrm{CB}, \mathrm{LP}}\right)$ is controlled using the sidestream flowrate (SLP);

Table 4

Loss for each disturbance

\begin{tabular}{|c|c|c|c|c|}
\hline \multirow[t]{2}{*}{ Control variable, $c$} & \multicolumn{2}{|c|}{ Loss $(\%)$ (feedrate disturbance) } & \multicolumn{2}{|c|}{ Loss (\%) (composition disturbance) } \\
\hline & $F+20 \%$ & $F-20 \%$ & $z_{\mathrm{F}, \mathrm{B}}+0.1$ & $z_{\mathrm{F}, \mathrm{B}}-0.1$ \\
\hline$P_{\mathrm{HP}}$ & 2.50 & 0.95 & 21.94 & 71.70 \\
\hline$L_{\mathrm{T}, \mathrm{HP}} / F$ & 0.02 & 0.01 & 0.99 & Infeasible \\
\hline$D_{\mathrm{HP}} / F$ & $\mathbf{0 . 0 3}$ & 0.02 & 1.3 & 1.47 \\
\hline$x_{\mathrm{BD}, \mathrm{HP}}$ & 0.02 & 0.06 & 26.96 & 38.71 \\
\hline$Q_{\mathrm{B}, \mathrm{HP}}$ & Infeasible & 24.55 & Infeasible & 2.44 \\
\hline$D_{\mathrm{HP}}$ & 8.40 & 19.14 & 1.33 & 1.41 \\
\hline$B_{\mathrm{HP}}$ & 20.80 & 28.11 & 1.33 & 1.41 \\
\hline$Q_{\mathrm{B}, \mathrm{HP}} / F$ & Infeasible & Infeasible & Infeasible & Infeasible \\
\hline$\widetilde{B}_{\mathrm{HP}} / \boldsymbol{F}$ & $\mathbf{0 . 0 3}$ & 0.02 & 1.30 & 1.47 \\
\hline$x_{\mathrm{BB}, \mathrm{HP}}$ & 0.02 & 0.14 & 40.92 & Infeasible \\
\hline$L_{\mathrm{T}, \mathrm{HP}}$ & Infeasible & 1.99 & 0.97 & Infeasible \\
\hline$T_{4, \mathrm{HP}}$ & 3.88 & 1.09 & 26.49 & Infeasible \\
\hline$x_{\mathrm{AB}, \mathrm{HP}}$ & Infeasible & Infeasible & Infeasible & Infeasible \\
\hline
\end{tabular}




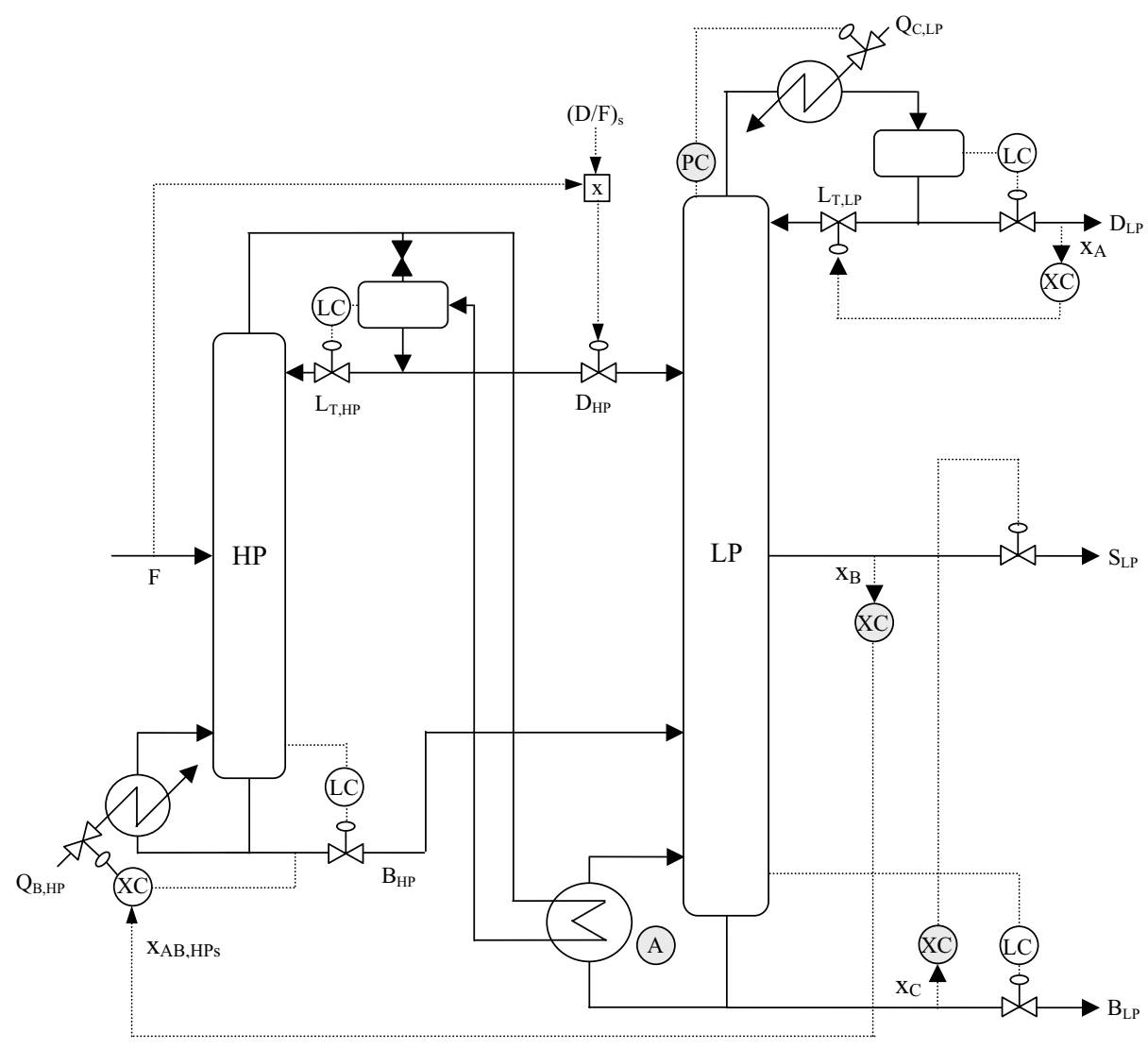

Fig. 3. Proposed control structure for the integrated prefractionator arrangement.

- the sidestream product purity $\left(x_{\mathrm{B}}=x_{\mathrm{BS}, \mathrm{LP}}\right)$ is controlled using the boilup in the HP column. To allow for local disturbance rejection this has been implemented in a cascade scheme, where the boilup is used to control the composition $x_{\mathrm{AB}, \mathrm{HP}}$ in the bottom of the HP column, and the setpoint for this bottom composition $\left(x_{\mathrm{AB}, \mathrm{HPs}}\right)$ is used as an independent variable to control the sidestream purity in the LP column $\left(x_{\mathrm{B}}=x_{\mathrm{BS}, \mathrm{LP}}\right)$;

- the pressure in the LP column is controlled using the condensation rate in the LP condenser $\left(Q_{\mathrm{C}, \mathrm{LP}}\right)$;

- the maximum area in the integrated reboiler/condenser is used (not actual control loop, bypass valve is fully closed).

(III) "Self-optimizing" loops (two)

- the distillate product purity $\left(x_{\mathrm{A}}=x_{\mathrm{AD}, \mathrm{LP}}\right)$ is controlled using the reflux flowrate $\left(L_{\mathrm{T}, \mathrm{LP}}\right)$;

- the flow ratio $D_{\mathrm{HP}} / F$ is controlled at a constant setpoint (self-optimizing loop).

\section{Dynamic simulations}

Dynamic simulations have been carried out in order to verify the proposed control structure in Fig. 3, where the ratio $D_{\mathrm{HP}} / F$ is used as a self-optimizing variable. Figs. 4-6 show the response in the product compositions when the system was subject to the following disturbances:

- $5 \%$ increase in the feedrate $F$ (Fig. 4);

- 0.05 increase in the middle component feed fraction (Fig. 5);

- 0.05 decrease in the middle component feed fraction (Fig. 6).

For all cases the disturbances have been made at time of $10 \mathrm{~h}$.

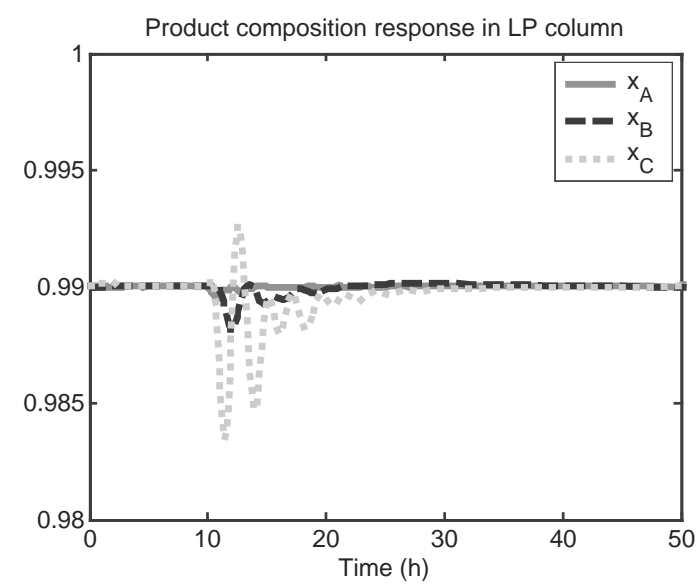

Fig. 4. Response to disturbance in feed flowrate $F+5 \%$ at time $=10 \mathrm{~h}$. 


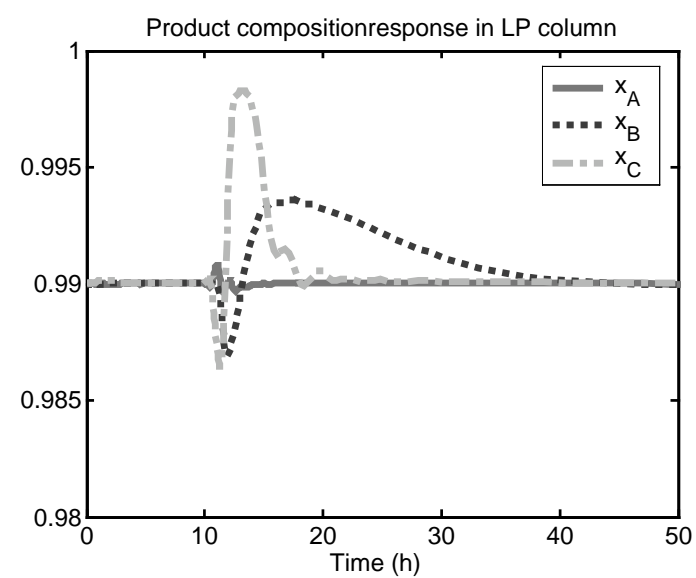

Fig. 5. Response to disturbance in feed composition, $z_{\mathrm{F}}=[0.175$ $0.650 .175]$ at time $=10 \mathrm{~h}$.

The results from the dynamic simulations show that the proposed control system works and that the integrated prefractionator arrangement is controllable, but that the system is quite sensitive to disturbances. If large disturbances are to be expected then other control schemes should also be investigated.

From the figures it can be seen that the distillate composition control is very fast and with small setpoint deviation. The sidestream has a slower response, as expected, since this is normally the case for the sidestream and in addition as this loop is the slow cascade loop. The bottom concentration response is the poorest with the largest setpoint deviation. If it is important to keep the concentration close to the setpoint then other alternatives may be investigated. The problem could be alleviated by, for example, using a product tank, which will average out the variations in product composition. Another alternative, which would give a faster response, would be to use an auxiliary reboiler to control the bottom composition. This however, would result in a higher energy demand and as the main objective in this study has been on energy saving this option has not been considered.

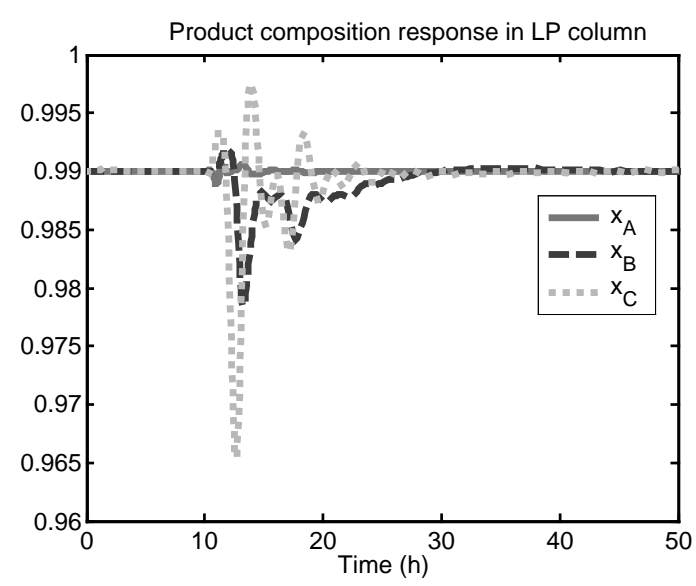

Fig. 6. Response to disturbance in feed composition, $z_{\mathrm{F}}=[0.125$ $0.750 .125]$ at time $=10 \mathrm{~h}$.

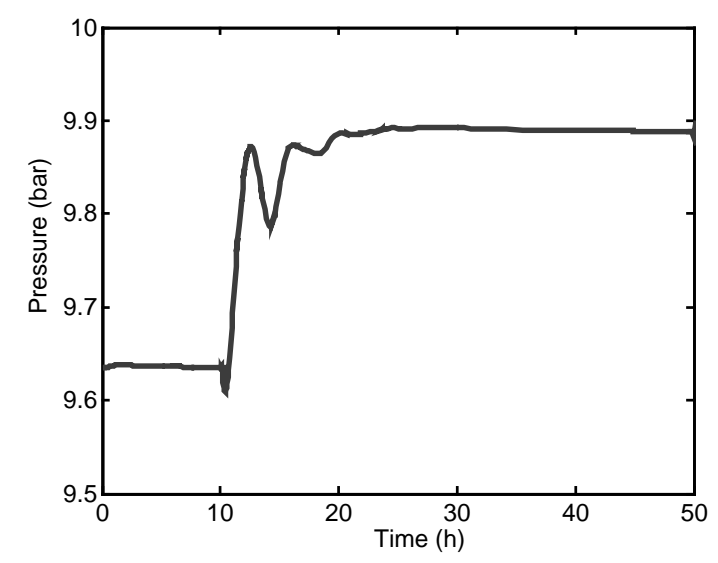

Fig. 7. Response of pressure in top of HP column response to a $5 \%$ increase in feed flowrate at time $=10 \mathrm{~h}$.

According to the results from the optimization the pressure in the HP column has been kept floating. A typical pressure response in the top of the HP column can be seen in Fig. 7. The disturbance is a $5 \%$ increase in the feed flowrate.

Two other structures with different control for the bottom composition were also tested:

- Alternative structure 1: The bottom composition is controlled in cascade, with a temperature below the sidestream controlled in an inner loop using the sidestream. The temperature setpoint is then used to control the bottom composition.

- Alternative structure 2: The boilup in the HP column is used to control the bottom composition in the LP column. For this scheme the bottom composition in the HP column was not controlled and the sidestream flowrate was used to control the sidestream composition.

None of these alternative structures showed improvement to the proposed structure. Structure 1 was controllable and worked reasonably well, but alternative 2 was very slow and did not work well.

\section{Discussion}

When considering heat-integrated distillation columns it is important that after the first initial screening of alternatives that a more rigorous analysis is performed. The results will often be on a case to case basis. For instance, for multi-effect columns the temperature range of the utilities is increased. For the system studied this means that the heat supplied to the HP column must be at a higher temperature than if the system was operating at a lower pressure. This may, for some cases, be within the available utilities on some plants whereas on other plants it may not be acceptable.

The identification and selection of the disturbances may also vary from case to case and this will influence the results. For instance, it may be that composition disturbances are not expected. There may then be variables other than 
the flow ratio that can be used for the self-optimizing control loop. There may also be other disturbances that should be considered, e.g. disturbances in the utilities, such as the steam pressure, or disturbances in the feed quality (vapour fraction).

As described earlier the set of the active constraints in the system may also change.

The list of control variable tested is not extensive and further variables should be tested. An alternative method can also be used where the optimal combination of control variables is found (giving no loss) (Alstad \& Skogestad, 2002).

Finally, in this study we have only considered the forward-integrated arrangement in Fig. 1. The backward integrated scheme with the prefractionator operating at the low pressure and the heat supplied to the main column has similar energy savings, but will otherwise be quite different from an operation point of view. For example, the temperature at the top of the LP column will be higher, assuming that the LP column is operated at 1 bar. The best self-optimizing variable is also likely to change. We found in our study of the forward integrated prefractionator arrangement that the control of the bottom product was somewhat poorer (e.g. see Fig. 6). The backward integrated scheme is potentially better from this point of view, since the boilup in the main column can be used for control purposes.

\section{Conclusions}

The work presented shows that a heat-integrated prefractionator arrangement has very large energy savings compared with conventional direct or indirect split columns. The focus of the work has been on finding good control variables that will ensure that these promised energy savings are achieved in practice during operation.

The method of self-optimizing control is used to find the best control variables. For the prefractionator arrangement there is a total of eleven degrees of freedom with six DOF available for optimization when variables with no-steady state effects have been excluded and the duties of the two columns are matched. From the optimization it was found that there are two degrees of freedom left for control variables had to be found. The active constraints $\left(P_{\mathrm{LP}}, x_{\mathrm{BS}, \mathrm{LP}}\right.$, $\left.x_{\mathrm{CB}, \mathrm{LP}}, A\right)$ were used to implemented active constraint control. Also, it was decided to control the top product purity $\left(x_{\mathrm{AD}, \mathrm{LP}}\right)$ in the LP column as this gave very low loss in the objective function. A comparison of economic loss was then used to find the last self-optimizing control variable that will keep the system close to the optimum when there are disturbances. The ratio of the distillate flow to the feed rate was found to have good self-optimizing properties. Dynamic studies where the $D_{\mathrm{HP}} / F$ scheme was implemented showed that the system is controllable, but that the sidestream and bottom stream composition responses are slow and that the system is sensible to disturbances.

For the selected feed composition the forward-integrated prefractionator arrangement uses only around 30\% of the energy required for the direct or indirect split arrangement. When including the average loss of $4.3 \%$ when controlling the distillate to feed ratio the forward-integrated prefractionator arrangement will use $31.3 \%$ of the energy required for the direct or indirect split arrangement.

\section{References}

Alstad, V., \& Skogestad, S. (2002). Robust operation by controlling the right variable combination. AIChE Annual Meeting, Paper $247 \mathrm{~g}$, Indianapolis, 3-8 November 2002.

Bildea, C. S., \& Dimian, A. C. (1999). Interaction between design and control of a heat-integrated distillation system with prefractionator. Transactions of IChemE, 77(Part A), 597-608.

Cheng, H. C., \& Luyben, W. (1985). Heat-integrated distillation columns for ternary separations. Industrial and Engineering Chemistry Process Design Development, 24, 707-713.

Christiansen, A. C. (1997). Studies on optimal design and operation of integrated distillation arrangements. Ph.D. Thesis, Norwegian University of Science and Technology.

Ding, S. S., \& Luyben, W. (1990). Control of a heat-integrated complex distillation configuration. Industrial and Engineering Chemistry Research, 29, 1240-1249.

Emtir, M., Rév, E., \& Fonyó, Z. (2001). Rigorous simulation of energy integrated and thermally coupled distillation schemes for ternary mixture. Applied Thermal Engineering, 21, 1299-1317.

Emtir, M., Rév, E., \& Fonyó, Z. (2003). Economic and controllability investigation and comparison of energy integrated distillation schemes. Chemical and Biochemical Engineering Quarterly, 17(1), 31-42.

Engelien, H. K. (2004). Process integration applied to the design and operation of distillation columns. Ph.D. Thesis, Norwegian University of Science and Technology.

Halvorsen, I. J. (1999). Optimal operation of Petlyuk distillation: Steady-state behavior. Journal of Processing Control, 9, 407424.

Hewitt, G., Quarini, J., \& Morell, M. (1999, October 21). More efficient distillation. The Chemical Engineer.

King, C. J. (1980). Separation processes. New York: McGraw-Hill.

Skogestad, S. (2000). Plantwide control: The search for the self-optimizing control structure. Journal of Processing Control, 10, 487-507. 